УДК 621.391

\section{ДИНАМИЧЕСКАЯ МОДЕЛЬ УПРАВЛЕНИЯ ОЧЕРЕДЯМИ НА ИНТЕРФЕЙСЕ МАРШРУТИЗАТОРА ТЕЛЕКОММУНИКАЦИОННОЙ СЕТИ}

ЛЕБЕДЕНКО Т.Н., СИМОНЕНКО А.В.

Предлагается динамическая модель управления очередями. Новизна предложенной модели заключается в учете динамики изменения состояния интерфейса маршрутизатора телекоммуникационной сети (ТКС) при распределении сетевого ресурса. Динамические свойства модели описываются нелинейными дифференциальными уравнениями состояния сети, основанными на стационарной точечной аппроксимации нестационарного потока (The Pointwise Stationary Fluid Flow Approximation, PSFFA).

Ключевые слова: динамическая модель; очередь; потоки; распределение ресурсов; качество обслуживания.

Key words: dynamic model, queue; flow; resource allocation; quality of service.

\section{1. Введение}

Совершенствование средств обеспечения качества обслуживания QoS (Quality of Service, QoS) потоков пользователей является одним из актуальных направлений развития современных телекоммуникационных сетей. Важное место среди подобных средств занимают механизмы управления очередями (Queue Management), позволяющие эффективно управлять буферными и (или) канальными ресурсами ТКС и непосредственно влиять на количественные значения таких показателей качества обслуживания как средняя задержка, джиттер, вероятность потерянных пакетов без существенной модернизации уже существующей сети [1-5].

Анализ известных решений в области управления буферными и канальными ресурсами ТКС [6-12] показал, что результаты работы применяемых математических моделей были получены, как правило, для установившегося режима работы системы. Ввиду того, что состояние ТКС может изменяться во времени вследствие нестационарной активности пользователей или типов применяемых сервисов, а также перегрузок или полного отказа в обслуживании отдельных элементов системы, то важным и необходимым является учет фактора времени при оценке ее состояния и расчете значений показателей качества обслуживания.

Таким образом, актуальной представляется задача разработки и усовершенствования динамических моделей и методов управления очередями, позволяющих обеспечить согласованное решение задач по управлению очередями с учетом динамики самого процесса управления и требований к качеству обслуживания.
2. Динамическая модель управления очередями на интерфейсе маршрутизатора ТКС

В основу предложенной модели были положены результаты, полученные в работах $[5,9,11,12]$. В табл.1 приведен перечень основных обозначений, используемых для описания динамической модели управления очередями на интерфейсе маршрутизатора телекоммуникационной сети.

Таблица 1

\begin{tabular}{|c|c|}
\hline M & $\begin{array}{l}\text { Количество потоков, поступающих на вход } \\
\text { интерфейса маршрутизатора ТКС }\end{array}$ \\
\hline $\mathrm{N}$ & $\begin{array}{l}\text { Количество очередей, организованных на } \\
\text { интерфейсе маршрутизатора ТКС }\end{array}$ \\
\hline $\mathrm{a}_{\mathrm{i}}(\mathrm{t})$ & $\begin{array}{l}\text { Средняя интенсивность і -го потока в момент } \\
\text { времени t }\end{array}$ \\
\hline $\mathrm{a}_{\mathrm{j}}^{\sum_{\mathrm{N}}(\mathrm{t})}$ & $\begin{array}{l}\text { Суммарная интенсивность потоков, } \\
\text { направленных на обслуживание в j-ю оче- } \\
\text { редь в момент времени t }\end{array}$ \\
\hline $\mathrm{b}_{\mathrm{j}}$ & $\begin{array}{l}\text { Пропускная способность интерфейса, } \\
\text { выделенная для обслуживания пакетов из j-й } \\
\text { очереди }\end{array}$ \\
\hline b & $\begin{array}{l}\text { Общая пропускная способность интерфейса, } \\
\text { на котором организуются очереди }\end{array}$ \\
\hline $\mathrm{k}_{\mathrm{i}}^{\Pi}$ & Значение класса каждого і -го потока \\
\hline $\mathrm{k}_{\mathrm{j}}^{\mathrm{o}}$ & Значение класса каждой j-й очереди \\
\hline$\overline{n_{j}}(t)$ & $\begin{array}{l}\text { Значение средней длины } \mathrm{j} \text {-й очереди в } \\
\text { момент времени t }\end{array}$ \\
\hline $\mathrm{n}_{\mathrm{j}}^{0}$ & $\begin{array}{l}\text { Значение средней длины } \mathrm{j} \text {-й очереди в } \\
\text { нулевой момент времени } \\
\end{array}$ \\
\hline $\mathrm{n}_{\mathrm{j}}^{\max }$ & Максимальная длина j-й очереди \\
\hline$\beta$ & $\begin{array}{l}\text { Зерхний динамический управляемый порог } \\
\text { агруженности очередей по их длине }\end{array}$ \\
\hline
\end{tabular}

В рамках предложенной модели условимся, что количество поступающих на вход интерфейса потоков пакетов $\mathrm{M}$ превышает число поддерживаемых на нем очередей $\mathrm{N}$ и имеет место неравенство $\mathrm{M}>\mathrm{N}$.

Для удобства последующего изложения условимся, что значение класса каждого i -го потока $\mathrm{k}_{\mathrm{i}}^{\Pi}$, как и значение класса каждой $\mathrm{j}$-й очереди $\mathrm{k}_{\mathrm{j}}^{0}$, нормируется в пределах от единицы до десяти и является безразмерной величиной. Поток с наивысшим значением класса, равным десяти, т.е. $\mathrm{k}_{\mathrm{i}}^{\Pi}=10$, определим как приоритетный поток. Тогда чем меньше класс, тем значение $\mathrm{k}_{\mathrm{i}}^{\text {п }}$ будет ближе к единице.

Пусть пакеты, поступившие в соответствии с содержанием актуальной таблицы маршрутизации на 
интерфейс маршрутизатора ТКС, должны распределиться между $\mathrm{N}$ очередями при расчете множества переменных первого типа $x_{i, j}(t)(i=1, M, j=1, N)$, каждая из которых определяет долю i -го потока, направленного на обслуживание в $\mathrm{j}$ - ю очередь в момент времени $\mathrm{t}$.

Для обеспечения приблизительно равного качества обслуживания пакетов одного и того же потока целесообразным является их обработка в рамках одной из очередей. Тогда множество переменных $\mathrm{x}_{\mathrm{i}, \mathrm{j}}(\mathrm{t})$ определим как булевые переменные:

$$
x_{i, j}(t) \in\{0,1\},(i=\overline{1, M}, j=\overline{1, N}) .
$$

На переменные $\mathrm{x}_{\mathrm{i}, \mathrm{j}}(\mathrm{t})$ также будет накладываться условие сохранения потока на интерфейсе маршрутизатора ТКС, гарантирующее, что все пакеты i -го потока (без потерь) будут направлены в одну из j-х очередей, организованных на рассматриваемом интерфейсе в момент времени $\mathrm{t}$ :

$$
\sum_{j=1}^{N} x_{i, j}(t)=1,(i=\overline{1, M}) \text {. }
$$

В рамках решения задачи Resource Allocation, связанной с выделением пропускной способности интерфейса каждой $\mathrm{j}$-й очереди, необходимо рассчитать множество переменных второго типа $-b_{j}$ :

$$
\sum_{j=1}^{N} b_{j} \leq b, 0<b_{j}, \quad(j=\overline{1, N}) .
$$

Выполнение условия (3) позволит корректно распределить пропускную способность (ПС) интерфейса между отдельными очередями.

Для обеспечения управляемости процессом предотвращения перегрузки определим суммарную интенсивность i-x потоков, направленных на обслуживание в j-ю очередь в момент времени $\mathrm{t}$ :

$$
\mathrm{a}_{\mathrm{j}}(\mathrm{t})=\sum_{\mathrm{i}=1}^{\mathrm{M}} \mathrm{a}_{\mathrm{i}}(\mathrm{t}) \mathrm{x}_{\mathrm{i}, \mathrm{j}}(\mathrm{t}), \quad(\mathrm{i}=\overline{1, \mathrm{M}}, \mathrm{j}=\overline{1, N}) .
$$

Тогда условие обеспечения управляемости процессом предотвращения перегрузки примет вид:

$$
\mathrm{a}_{\mathrm{j}}(\mathrm{t})<\mathrm{b}_{\mathrm{j}},(\mathrm{j}=\overline{1, \mathrm{~N}}) .
$$

Удовлетворение условия (5) гарантирует, что суммарная интенсивность потоков, направленных на обслуживание в j-ю очередь в момент времени $t$, не превысит пропускную способность интерфейса, выделенную данной очереди.

В целях предотвращения перегрузки очереди по ее длине (в пакетах) дополним условия (1)-(5) нелинейным ограничением вида:

$$
\overline{n_{j}}(t) \leq n_{j}^{\max },(j=\overline{1, N}),
$$

где значения средней длины $\mathrm{j}$-й очереди в момент времени $t-\overline{n_{j}}(t)$ будут зависеть от характеристик потоков, формирующих данную очередь, от поддерживаемой дисциплины обслуживания, от выделенной данной очереди пропускной способности интерфейса и от момента времени $\mathrm{t}[9,11,12]$.

\section{3. Особенности расчета средней длины очереди на интерфейсе маршрутизатора ТКС}

Ввиду того, что оценка состояния интерфейса и последующее управление очередями являются процессами реального времени, то актуальным и необходимым видится использование в разрабатываемой модели математического аппарата, учитывающего динамику изменения его состояния во времени. Так, в научных исследованиях посвященных данной проблематике [5-12], предлагается примененять аппарат теории массового обслуживания, где в зависимости от используемой дисциплины обслуживания становится возможным оценить влияние состояния интерфейса (пропускной способности, загруженности и максимальной длины очереди) на средний размер буфера и, следовательно, численные значения показателей QoS.

Анализ $[5,9,11,12]$ показал, что для стационарного режима работы интерфейса маршрутизатора ТКС, т.е. режима, при котором все переходные процессы, связанные с изменением его состояния, окончены, расчет средней длины очереди (6) может быть описан следующими аналитическими зависимостями:

$\mathrm{M} / \mathrm{M} / 1$ :

$$
\overline{\mathrm{n}_{\mathrm{j}}}=\frac{\rho}{1-\rho},
$$

$\mathrm{M} / \mathrm{D} / 1$ :

$$
\overline{n_{j}}=\frac{\rho}{1-\rho}-\frac{\rho^{2}}{2(1-\rho)}
$$

где $\rho=\frac{\mathrm{a}_{\mathrm{j}}}{\mathrm{b}_{\mathrm{j}}}-$ коэффициент использования пропускной способности, выделенный для каждой jочереди.

Применение данных зависимостей (7)-(8) и отсутствие учета фактора времени в ходе оценки состояния интерфейса может привести к неадекватному и нерациональному расчету значений показателей качества обслуживания. В этой связи актуальным является переход к математическому аппарату, позволяющему учесть динамический характер изменений, которые происходят на интерфейсах маршрутизаторов в масштабах реального времени диапазона секунд.

Стоит отметить, что для описания динамики изменения состояния интерфейса во времени исполь- 
зуют различные типы математических аппроксимаций, из которых наиболее эффективной с точки зрения адекватности, наглядности и применимости, на наш взгляд, является стационарная точечная аппроксимация нестационарного потока (The Pointwise Stationary Fluid Flow Approximation, PSFFA) [9, 11, 12]. Согласно PSFFA значения средней длины очереди в момент времени $\mathrm{t}$ (6) будут описываться нелинейными дифференциальными уравнениями состояния сети:

$$
\begin{gathered}
M(t) / M(t) / 1: \frac{d \overline{n_{j}}(t)}{d t}=a_{j}(t)-b_{j}\left(\frac{\overline{n_{j}}(t)}{\overline{n_{j}}(t)+1}\right), \\
M(t) / D(t) / 1: \frac{d \overline{n_{j}}(t)}{d t}=a_{j}(t)-b_{j}\left[\left(\overline{n_{j}}(t)+1\right)-\sqrt{\overline{n_{j}}(t)^{2}+1}\right] .
\end{gathered}
$$

Тогда при условии моделирования работы интерфейса, например, одноканальной системой массового обслуживания с нестационарным пуассоновским потоком и нестационарным экспоненциальным временем обслуживания $(\mathrm{M}(\mathrm{t}) / \mathrm{M}(\mathrm{t}) / 1)$, выражение для оценки средней длины очереди $\overline{\mathrm{n}_{\mathrm{j}}}(\mathrm{t})$ примет следующий вид:

$$
\begin{aligned}
& \overline{n_{j}}(t)=-\left(a_{j}(t)+b_{j} \cdot W\left(0,-\exp \left(-\left(a_{j}(t)+\left(a_{j}(t)-\right.\right.\right.\right.\right. \\
& \left.-b_{j}\right)^{2} \cdot\left(t-\left(b _ { j } \cdot \left(\operatorname { l n } \left(\operatorname { e x p } \left(-\left(a_{j}(t)+n_{j}^{0} \cdot\left(a_{j}(t)-b_{j}\right)\right) /\right.\right.\right.\right.\right. \\
& \left.\left.\left.\left./ b_{j}\right) \cdot\left(a_{j}(t)+n_{j}^{0} \cdot\left(a_{j}(t)-b_{j}\right)\right)\right)+a_{j}(t) / b_{j}\right)\right) / \\
& \left.\left.\left.\left./\left(a_{j}(t)-b_{j}\right)^{2}\right)\right) / b_{j}\right) /\left(b_{j}\right)\right) /\left(a_{j}(t)-b_{j}\right)
\end{aligned}
$$

где $\mathrm{W}-\quad$ функция Ламберта; $\exp -$ экспоненциальная функция.

Использование аппроксимации PSFFA позволяет отслеживать текущее значение средней длины очереди на интерфейсе маршрутизатора, а также прогнозировать ее значение на других временных интервалах.

В дополнение к условиям (1)-(6) в структуру предлагаемой динамической модели введем еще одно дополнительное нелинейное ограничение, позволяющее обеспечить сбалансированную загрузку создаваемых на интерфейсе маршрутизатора очередей и отвечающее концепции Traffic Engineering Queues [6-12]:

$$
\mathrm{k}_{\mathrm{j}}^{\mathrm{o}} \overline{\mathrm{n}_{\mathrm{j}}}(\mathrm{t}) \leq \beta \mathrm{n}_{\mathrm{j}}^{\max }, \quad(\mathrm{j}=\overline{1, N}) .
$$

Физический смысл условия (12) заключается в том, чтобы создаваемые на интерфейсе маршрутизатора очереди загружались сбалансированно. При этом чем выше значение класса очереди $\mathrm{k}_{\mathrm{j}}^{\mathrm{o}}$ тем меньшую длину $\overline{\mathrm{n}_{\mathrm{j}}}(\mathrm{t})$ она должна иметь.

\section{4. Формулировка критерия оптимальности ре- шения по управлению очередями}

Согласно сформулированным условиям и ограничениям рассматриваемой динамической модели, расчет искомых переменных целесообразно осуществить в ходе решения следующей оптимизационной задачи:

$$
\min _{x, b, \beta} F \text {. }
$$

Тогда целевую функцию $\mathrm{F}$ представим линейной формой вида:

$$
F=\sum_{i=1}^{M} \sum_{j=1}^{N} h_{i, j}^{x} x_{i, j}+\sum_{j=1}^{N} h_{j}^{b} b_{j}+h^{\beta} \beta,
$$

где $\mathrm{h}_{\mathrm{i}, \mathrm{j}}^{\mathrm{x}}$ - условная стоимость (метрика) обслуживания пакетов $i$-го потока с помощью $\mathrm{j}$-й очереди; $\mathrm{h}_{\mathrm{j}}^{\mathrm{b}}$ - условная стоимость (метрика) выделения единицы пропускной способности интерфейса пакетам из j-й очереди; $\mathrm{h}^{\beta}-$ условная стоимость реализации процесса балансировки длин очередей на интерфейсе маршрутизатора.

C точки зрения физического смысла сформулированной задачи (13), расчет обозначенных управляющих переменных $\mathrm{x}_{\mathrm{i}, \mathrm{j}}, \mathrm{b}_{\mathrm{j}}$ и $\boldsymbol{\beta}$ должен приводить к минимизации суммарной стоимости использования сетевых ресурсов, где первое слагаемое отвечает за порядок использования буфера очереди (задача Congestion Management), второе - за пропускную способность интерфейса (задача Resource Allocation), а третье - за качество решения всех поставленных задач.

Ввиду того, что критерием направленности тех или иных потоков в соответствующие им очереди является максимальная соизмеримость классов потоков $\mathrm{k}_{\mathrm{i}}^{\Pi}$ и классов очередей $\mathrm{k}_{\mathrm{j}}^{\mathrm{o}}$, то метрику $\mathrm{h}_{\mathrm{i}, \mathrm{j}}^{\mathrm{x}}$, отвечающую за распределение потоков по очередям в момент времени tи их агрегирование при выполнении условия $\mathrm{M}>\mathrm{N}$, будем рассчитывать по формуле:

$$
\mathrm{h}_{\mathrm{i}, \mathrm{j}}^{\mathrm{x}}=\mathrm{w}_{\mathrm{x}}^{\mathrm{b}}\left(\mathrm{k}_{\mathrm{i}}^{\Pi}-\mathrm{k}_{\mathrm{j}}^{\mathrm{o}}\right)^{2}
$$

где $\mathrm{w}_{\mathrm{x}}^{\mathrm{b}}$ - регулировочный параметр, влияющий на конечное численное значение целевой функции (13). 


\section{5. Исследования процессов управления очере- дями с использованием предложенной динами- ческой модели}

Продемонстрируем пример решения задачи управления очередями на интерфейсе маршрутизатора ТКС с применением динамической модели. В табл.2 приведены исходные данные и результаты расчета для двадцати пяти потоков ( $\mathrm{M}=25)$, десяти очередей $(\mathrm{N}=10)$, пропускной способности интерфейса $(b=1001 / c)$, регулировочного параметра $\left(\mathrm{w}_{\mathrm{x}}^{\mathrm{b}}=100\right)$ и временного интервала $\mathrm{t}=2 \mathrm{c}$.

В ходе моделирования количество потоков ( $\mathrm{M})$ и количество очередей ( N ) полагалось известным.

Классы очередей $\left(\mathrm{k}_{\mathrm{j}}^{\mathrm{o}}\right)$ распределялись детерминировано в пределах от единицы до десяти. Классы потоков $\left(k_{i}^{\Pi}\right)$ были определены по случайному за- кону распределения и также варьировались в диапазоне от 1 до 10 включительно.

Исследования процессов управления очередями на интерфейсе маршрутизатора ТКС проводилось в рамках двух моделей. Так, модель М1 описывала динамический режим работы интерфейса, где значения средней длины очереди были получены посредствам аппроксимации PSFFA для одноканальной системы массового обслуживания с нестационарным пуассоновским потоком и нестационарным экспоненциальным временем обслуживания $\mathrm{M}(\mathrm{t}) / \mathrm{M}(\mathrm{t}) / 1$ (11). Модель М2 определяла стационарный режим работы интерфейса, в котором значения средней длины очереди были описаны одноканальной системой массового обслуживания с пуассоновским входным потоком и экспоненциальным распределением времени обслуживания $\mathrm{M} / \mathrm{M} / 1$ (7).

Таблица 2

\begin{tabular}{|c|c|c|c|c|c|c|c|c|c|c|}
\hline \multicolumn{3}{|c|}{ Характеристики потоков } & \multicolumn{8}{|c|}{ Параметры очередей } \\
\hline \multirow{2}{*}{$\mathrm{M}$} & \multirow{2}{*}{$\mathrm{k}_{\mathrm{i}}^{\Pi}$} & \multirow{2}{*}{$a_{i}(t)$} & \multirow{2}{*}{$\mathrm{N}$} & \multirow{2}{*}{$\mathrm{k}_{\mathrm{j}}^{\mathrm{o}}$} & \multirow{2}{*}{$\mathrm{n}_{\mathrm{j}}^{0}$} & \multirow{2}{*}{$a_{j}(t)$} & \multicolumn{2}{|c|}{ M1 } & \multicolumn{2}{|r|}{ M2 } \\
\hline & & & & & & & $b_{j}{ }^{\text {I }}$ & $\overline{n_{j}}(t)^{Д / n_{j}}$ & $\mathrm{~b}_{\mathrm{j}}^{\mathrm{c}}$ & $\overline{n_{j}}(t){ }^{c} / n_{j}^{c \max }$ \\
\hline 5 & 1,1900 & 3,0290 & \multirow[t]{3}{*}{1} & \multirow[t]{3}{*}{1} & \multirow[t]{3}{*}{0} & \multirow[t]{3}{*}{11,4128} & \multirow[t]{3}{*}{11,4128} & \multirow{3}{*}{$5,8301 / 100$} & \multirow[t]{3}{*}{11,4128} & \multirow[t]{3}{*}{$5,6014 / 100$} \\
\hline 18 & 1,3862 & 3,3971 & & & & & & & & \\
\hline 19 & 1,4929 & 4,9867 & & & & & & & & \\
\hline 4 & 1,6261 & 3,9427 & \multirow[t]{3}{*}{2} & \multirow[t]{3}{*}{2} & \multirow[t]{3}{*}{0} & \multirow[t]{3}{*}{9,8889} & \multirow[t]{3}{*}{9,8889} & \multirow[t]{3}{*}{$5,3683 / 90$} & \multirow[t]{3}{*}{10,2681} & \multirow[t]{3}{*}{$4,9414 / 90$} \\
\hline 10 & 2,2381 & 5,8700 & & & & & & & & \\
\hline 24 & 2,4352 & 0,0762 & & & & & & & & \\
\hline 22 & 2,5428 & 3,6405 & \multirow[t]{5}{*}{3} & \multirow[t]{5}{*}{3} & 0 & 20,4499 & 20,4499 & $8,0994 / 80$ & 21,9268 & $6,5580 / 80$ \\
\hline 12 & 2,5510 & 4,8461 & & & & & & & & \\
\hline 20 & 2,5751 & 5,9777 & & & & & & & & \\
\hline 1 & 2,7603 & 2,2399 & & & & & & & & \\
\hline 8 & 3,4039 & 3,7457 & & & & & & & & \\
\hline- & - & - & 4 & 4 & 0 & - & - & - & - & - \\
\hline 6 & 4,9836 & 2,2506 & 5 & 5 & 0 & 7,4198 & 7,4199 & $4,5389 / 60$ & 8,6106 & $3,4591 / 60$ \\
\hline 13 & 5,0596 & 4,8239 & & & & & & & & \\
\hline 17 & 5,4722 & 0,3453 & & & & & & & & \\
\hline 9 & 5,8527 & 3,5182 & 6 & 6 & 0 & 3,5182 & 3,5182 & $2,8823 / 50$ & 4,3313 & $2,2345 / 50$ \\
\hline 3 & 6,5510 & 1,6069 & 7 & 7 & 0 & 5,3000 & 6,8727 & $2,4289 / 40$ & 7,0863 & $2,3021 / 40$ \\
\hline 2 & 6,7970 & 1,2582 & & & & & & & & \\
\hline 14 & 6,9908 & 2,4349 & & & & & & & & \\
\hline 11 & 7,5127 & 1,8294 & 8 & 8 & 0 & 5,6649 & 9,0667 & $1,5940 / 30$ & 8,5743 & $1,8144 / 30$ \\
\hline 23 & 8,1428 & 3,0041 & & & & & & & & \\
\hline 21 & 8,4072 & 0,8314 & & & & & & & & \\
\hline 15 & 8,9090 & 3,6341 & 9 & 9 & 0 & 5,7917 & 11,9163 & $0,9446 / 20$ & 10,8112 & $1,1592 / 20$ \\
\hline 25 & 9,2926 & 2,1576 & & & & & & & & \\
\hline 16 & 9,5929 & 0,4855 & 10 & 10 & 0 & 5,8028 & 19,4546 & $0,4251 / 10$ & 16,9786 & $0,5248 / 10$ \\
\hline 7 & 9,5974 & 5,3173 & & & & & & & & \\
\hline
\end{tabular}

Согласно результатам моделирования, в рамках приведенного в табл.2 примера, удалось сократить число используемых очередей на 10\%. Четвертая очередь не была задействована ввиду отсутствия потоков с соизмеримыми для них классами. Таким образом, применение данной модели дало возможность использования лишь минимально необходимого числа очередей, тем самым позволяя сокра- 
тить возможные задержки, вносимые планировщиком пакетов.

Также определили, что значение верхнего динамического управляемого порога загруженности очередей для модели М2 со стационарным режимом работы интерфейса ( $\beta=0,5192)$ на $22 \%$ превышает значение $\beta$ относительно модели M1 с динамическим режимом работы интерфейса $(\beta=0,4251)$. По своему физическому смыслу параметр $\beta$ соответствует максимальному коэффициенту загруженности очередей, от значений которого зависят такие показатели QoS, как средняя задержка пакетов и их потеря [1-5].

В рамках данного примера аналогичного значения параметра $\beta$ для модели M2 удалось добиться лишь при увеличении пропусной способности интерфейса до 110 1/c.

\section{6. Выводы}

Рассмотрена динамическая модель управления очередями, базирующаяся на распределении сетевого ресурса. На основании множества классификационных признаков и требований к уровню QoS была произведена агрегация потов, позволяющая сократить число используемых очередей и вносимых планировщиком пакетов задержек, связанных с процессом формирования очередей, их анализом.

Расчетные решения, полученные в ходе использования предложенной модели, позволили обеспечить согласованное решение таких задач:

- распределение потоков пакетов между сформированными на интерфейсе маршрутизатора ТКС очередями;

- агрегирование потоков пакетов в соответствующие им очереди на основании соизмеримости их классов;

- распределение пропускной способности интерфейса между отдельными очередями (Resource Allocation).

Новизна модели заключалась в учете динамики изменения состояния интерфейса маршрутизатора ТКС при распределении сетевого ресурса. Динамические свойства модели описаны нелинейными дифференциальными уравнениями состояния сети, основанными на стационарной точечной аппроксимации нестационарного потока PSFFA.

Произведенный анализ показал, что использование предельных оценок (7)-(8) при расчете средней длины очереди (6) возможен лишь по окончанию всех переходных процессов, происходящих на интерфейсе маршрутизатора ТКС. В противном случае необходимым является использование более точной дифференциальной модели (9)-(10). Так, применение модели PSFFA позволило улучшить точность расчета средней длины очереди в зависимости от состояния интерфейса и выбранной дисциплины обслуживания и, в свою очередь, повлиять на численные показатели QoS.

Основываясь на результатах, полученных в ходе применения представленной динамической модели, удалось добиться экономии сетевого ресурса в диапазоне от 3,16 до $33 \%$ относительно стационарного режима работы интерфейса маршрутизатора ТКС. Предложенная модель может быть использована при решении задач анализа основных показателей качества обслуживания, а также при разработке перспективных моделей управления трафиком в современных мультисервисных сетях.

Литература: 1. Barreiros, M. QOS-Enabled Networks: Tools and Foundations / M. Barreiros, P. Lundqvist. Wiley: Wiley Series on Communications Networking \& Distributed Systems, 2nd Edition, 2016. 254p. 2. Szigeti, T. End-to-End QoS Network Design: Quality of Service for Rich-Media \& Cloud Networks / T. Szigeti, C. Hattingh, R. Barton, and K. Briley. 2nd Edition, Cisco Press, 2013. 1040 p. 3. Stallings, $W$. Foundations of modern networking: SDN, NFV, QoE, IoT, and Cloud. Addison-Wesley Professional, 2015. 544p. 4. Varma, S. Internet congestion control. Morgan Kaufmann, 2015. 263p. 5. Wang, P. Dynamics of Delay Differential Equations in Communications Networks: In the Framework of Active Queue Management. LAP Lambert Academic Publishing, 2009. 104p. 6. John, J. Priority Queuing Technique Promoting Deadline Sensitive Data Transfers in Router based Heterogeneous Networks / J. John, R. Balan // International Journal of Applied Engineering Research 12(15), 2017. P. 4899-4903. 7. Lemeshko, $O$. Researching and designing of the Dynamic adaptive queue balancing method on telecommunication network routers / O. Lemeshko, M. Semenyaka, O. Simonenko // Proceedings of XIIth CADSM 2013, 2013. P. 204-207. 8. Lemeshko, O. Results of the Dynamic Flow-Based Queue Balancing Model Research / O. Lemeshko, Ali S. Ali, M. Semenyaka // Modern Problems of Radio Engineering, Telecommunications and Computer Science (TCSET), 11th International Conference on. IEEE, 2012. P. 318-319. 9. Yeremenko, $O$. Investigation of queue utilization on network routers by the use of dynamic models / O. Yeremenko, T. Lebedenko, T. Vavenko and M. Semenyaka // In Problems of Infocommunications Science and Technology (PIC S\&T), Second International Scientific-Practical Conference on IEEE, 2015. P. 46-49. 10. Lemeshko, O. Dynamics analysis of multipath QoS-routing tensor model with support of different flows classes / O. Lemeshko, O. Yeremenko // Smart Systems and Technologies (SST), on. IEEE, 2016. P. 225-230. 11. Lebedenko, T. A queue management model on the network routers using optimal flows aggregation / T. Lebedenko, A. Simonenko, Fouad Abdul Razzaq Arif // Modern Problems of Radio Engineering, Telecommunications and Computer Science (TCSET), 13th Interna- 
tional Conference on. IEEE, 2016. P. 605-608. 12. Lebedenko, $T$. Research of influence flow characteristics to network routers queues utilization / T. Lebedenko, M. Ievdokymenko, Ali Salem Ali // Advanced Information and Communication Technologies: proceedings of 2015 1st International Conference (AICT'2015), 2015. P. 111-112.

Транслитерированный список литературы:

1. Barreiros, M. QOS-Enabled Networks: Tools and Foundations / M. Barreiros, P. Lundqvist. Wiley: Wiley Series on Communications Networking \& Distributed Systems, 2nd Edition, 2016. 254p.

2. Szigeti, T. End-to-End QoS Network Design: Quality of Service for Rich-Media \& Cloud Networks / T. Szigeti, C. Hattingh, R. Barton, and K. Briley. 2nd Edition, Cisco Press, 2013. 1040 p.

3. Stallings, $W$. Foundations of modern networking: SDN, NFV, QoE, IoT, and Cloud. Addison-Wesley Professional, 2015. 544p.

4. Varma, S. Internet congestion control. Morgan Kaufmann, 2015. 263p.

5. Wang, $P$. Dynamics of Delay Differential Equations in Communications Networks: In the Framework of Active Queue Management. LAP Lambert Academic Publishing, 2009. 104p.

6. John, J. Priority Queuing Technique Promoting Deadline Sensitive Data Transfers in Router based Heterogeneous Networks / J. John, R. Balan // International Journal of Applied Engineering Research 12(15), 2017. P. 4899-4903.

7. Lemeshko, $O$. Researching and designing of the Dynamic adaptive queue balancing method on telecommunication network routers / O. Lemeshko, M. Semenyaka, O. Simonenko // Proceedings of XIIth CADSM 2013, 2013. P. 204207.

8. Lemeshko, O. Results of the Dynamic Flow-Based Queue Balancing Model Research / O. Lemeshko, Ali S. Ali, M. Semenyaka // Modern Problems of Radio Engineering, Telecommunications and Computer Science (TCSET), 11th International Conference on. IEEE, 2012. P. 318-319.

9. Yeremenko, $O$. Investigation of queue utilization on network routers by the use of dynamic models / O. Yeremenko, T. Lebedenko, T. Vavenko and M. Semenyaka // In Problems of Infocommunications Science and Technology (PIC S\&T), Second International Scientific-Practical Conference on IEEE, 2015. P. 46-49.
10. Lemeshko, $O$. Dynamics analysis of multipath QoSrouting tensor model with support of different flows classes / O. Lemeshko, O. Yeremenko // Smart Systems and Technologies (SST), on. IEEE, 2016. P. 225-230.

11. Lebedenko, T. A queue management model on the network routers using optimal flows aggregation / T. Lebedenko, A. Simonenko, Fouad Abdul Razzaq Arif // Modern Problems of Radio Engineering, Telecommunications and Computer Science (TCSET), 13th International Conference on. IEEE, 2016. P. 605-608.

12. Lebedenko, $T$. Research of influence flow characteristics to network routers queues utilization / T. Lebedenko, M. Ievdokymenko, Ali Salem Ali // Advanced Information and Communication Technologies: proceedings of 2015 1st International Conference (AICT'2015), 2015. P. 111-112.

Поступила в редколлегию 12.02.2018 Рецензент: д-р техн. наук, проф. Баранник В.В.

Лебеденко Татьяна Николаевна, аспирантка кафедры инфокоммуникационной инженерии ХНУРЭ. Научные интересы: управление трафиком, качество обслуживания, отказоустойчивая маршрутизация. Адрес: Украина, 61166, Харьков, пр. Науки, 14, тел. +380577021320, еmail: tetiana.lebedenko@gmail.com.

Симоненко Александр Викторович, научный сотрудник научного центра, Харьковский национальный университет воздушных сил им. Ивана Кожедуба. Научные интересы: NGN, управление трафиком, качество обслуживания. Адрес: Украина, 61023, Харьков, ул. Сумская, 77/79 тел.+380577002165, e-mail:28186@ukr.net.

Tetiana Lebedenko, postgraduate student of the Department of Infocommunication Systems, Kharkiv National University of Radio Electronics. Research interests: Traffic Management, Quality of Service, Fault-Tolerant Routing. Address: Ukraine, 61166, Kharkiv, Nauka Ave., 14, Phone/fax: +380577021320, e-mail: tetiana.lebedenko@gmail.com.

Oleksandr Simonenko, researcher of the Scientific Center, Ivan Kozhedub Kharkiv National Air Force University. Research interests: NGN, Traffic Management, Quality of Service. Address: Ukraine, 61023, Kharkiv, Sumska St., 77/79, Phone/fax: +380577002165, e-mail:28186@ukr.net. 